\title{
МАТЕМАТИЧНА МОДЕЛЬ ДИНАМІКИ УСТАНОВКИ ПІДГОТОВКИ ВУГЛЕКИСЛОГО ГАЗУ У ТЕХНОЛОГІЧНОМУ ПРОЦЕСІ ВИРОБНИЦТВА КАРБАМІДУ
}

Definition of time of transition process by means of the modified number of homochronicity on the example of heating of bodies

А.И.Брунеткин, А.В.Гусак

Copyright (C) 2014 by author and the journal “Automation technological and business - processes”. This work is licensed under the Creative Commons Attribution International License (CC BY). http://creativecommons.org/licenses/by/4.0/

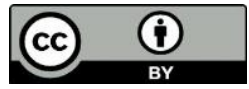

ONAFT

Open Access

\begin{abstract}
Анотация
Приведена приближенная модель в сосредоточенной постановке, описывающая процесс нагрева бесконечной пластины, цилиндра, шара, позволяющая определить момент его окончания. Полученные результаты сравнены с известными точными решениями на основе распределенной модели. Показана их высокая точность при изменении критерия Био в диапазоне 0,005 - 1000. Для всех рассматриваемых форм тел результаты описаны с помощью единого выражения, в зависимости от одной переменной в форме числа подобия - модифицированного числа гомохронности.
\end{abstract}

Annotation

The approximate model in the concentrated statement describing process of heating of an infinite plate, the cylinder, a sphere, allowing to define the moment of its termination is given. The received results are compared to known exact decisions on the basis of the distributed model. Their high precision at change of criterion of Bio in range of 0,005 1000 is shown. For all considered shapes of bodies results are described by means of uniform expression, depending on one variable in the form of similarity number - the modified gomokhronnost.

Ключові слова

Нагрев тела, переходный процесс, модель в сосредоточенной постановке, критерий подобия, число подобия, число гомохронности.

\section{Введение}

При решении технических задач исследуются процессы, развивающиеся как во времени, так и в пространстве. Нахождение аналитического решения такого рода задач затруднительно, а чаще всего невозможно. Экспериментальные исследования и родственные, в определенном смысле, им численные методы (вычислительный эксперимент) позволяют получить решение задач различной сложности, но лишь в отдельных как временны’х, так и пространственных точках. Дискретный характер решений не позволяет выявить всех возможных взаимосвязей искомых величин в пространстве их вариаций. В этой ситуации используются упрощения, позволяющие получить аналитические решения при учете наиболее важных свойств исследуемых объектов. Чаще всего это происходит путем замены объектов с распределенными параметрами «сосредоточенными» моделями. 
Такой подход реализован, например, в классической теории автоматического управления (ТАУ).

Замену распределенной модели сосредоточенной можно произвести в случае:

- если некоторый процесс является равновесным по отношению к характерному масштабу времени сопряженного процесса. В этом случае в качестве параметра сосредоточенной модели принимается равновесное значение из распределенной модели;

- если известен в динамике характер изменения исследуемого параметра (профиль параметра) в пространстве исследуемого объекта. В этом случае в качестве параметра сосредоточенной модели может быть принято его среднеинтегральное значение. Такой подход реализован в [1], где модель записана с точностью до некоторого коэффициента, вычисляемого при простых формах исследуемого пространства, либо получаемого в процессе ограниченного количества простых экспериментов.

Постановка задачи

Профиль параметра чаще всего неизвестен т.к. и является целью решения задачи в «распределенной» постановке. Но существует группа задач, при решении которых можно обоснованно сделать предположение о характере изменения искомого параметра в пространстве исследуемого объекта. Этого оказывается достаточным для определения с некоторой погрешностью времени переходного процесса и достижения исследуемым объектом равновесного состояния. Проиллюстрируем возможность такого подхода на примере задачи нагрева-охлаждения тел простых форм: бесконечная пластина, бесконечный цилиндр, шар. Для этой задачи существует точное решение, что позволяет оценить величину погрешности приближенного решения.

В качестве искомого параметра рассматривается температура тела в процессе его нагрева. О степени завершенности процесса можно судить по величине отклонения температуры в центре тела от температуры окружающей среды. Исходные условия запишем в общепринятой постановке [2]:

- начало координат расположено в центре тела;

- до начала нагрева тело находилось в равновесном состоянии с окружающей средой и имело температуру $t_{0}$. В начальный момент температура окружающей среды скачком изменяется от $t_{0}$ до $t_{\text {срд }}=$ const ;

- все температуры рассматриваются в отклонениях относительно начальной температуры тела. В этом случае: $\vartheta_{\text {п }}=t_{\text {п }}-t_{0} ; \vartheta_{ц}=t_{ц}-t_{0} ; \vartheta_{\text {срд }}=t_{\text {срд }}-t_{0}=$ const $; \widehat{\vartheta}=\widehat{t}-t_{0}$.

- в качестве граничных приняты условия III рода как наиболее общие.

Известно, что процесс нагрева-охлаждения тел носит экспоненциальный характер. Положим, что профиль изменения температуры от $\vartheta_{\text {п }}$ до $\vartheta_{ц}$ в процессе нагрева хотя и изменяется по количественным параметрам, но сохраняется на качественном уровне, также носит экспоненциальный характер и может быть описан в виде

$$
\vartheta_{\mathrm{x}}=\vartheta_{ц}+\left(\vartheta_{\Pi}-\vartheta_{ц}\right) \cdot \exp (1-l / x) .
$$

Величины $\vartheta_{\text {п }}$ и $\vartheta_{\text {ц }}$ неизвестны и подлежат определению. Значение среднеинтегральной температуры может быть определено из соотношения

$$
\widehat{\vartheta}=\frac{1}{V_{\mathrm{T}}} \int_{0}^{l} \vartheta_{\mathrm{x}} S_{\mathrm{x}} d x,
$$

а среднеинтегральное расстояние $\hat{l}$ от начала координат, на котором эта температура условно реализуется

$$
\widehat{l}=\frac{1}{V_{\mathrm{T}}} \int_{0}^{l} x \cdot \exp (1-l / x) \cdot S_{\mathrm{x}} d x .
$$

Так, например, для шара $S_{\mathrm{x}}=4 \pi x^{2}$, а для пластины $S_{\mathrm{x}}=S_{\text {бок }}$ - площадь одной боковой стороны пластины.

Рассмотрим нагрев пластины. В силу ее симметрии учитывается нагрев только через одну боковую поверхность и половина толщины. В этом случае 


\section{СТУДЕНТСЬКА НАУКА}

$$
V_{\mathrm{T}}=S_{\text {бок }} \delta
$$

С учетом (1) выражение (2) можно записать в виде

$$
\widehat{\vartheta}=\frac{1}{S_{\text {бок }} \delta} \int_{0}^{\delta}\left[\vartheta_{\text {ц }}+\left(\vartheta_{\text {п }}-\vartheta_{\text {ц }}\right) \cdot \exp (1-\delta / x)\right] S_{\text {бок }} d x
$$

и после преобразований

$$
\widehat{\vartheta}=\frac{1}{\delta}\left[\vartheta_{\Perp} \delta+\left(\vartheta_{\Pi}-\vartheta_{\amalg}\right) \cdot \exp (1) \cdot \int_{0}^{\delta} \exp (-\delta / x) d x\right]
$$

или

$$
\widehat{\vartheta}=\frac{1}{\delta}\left\{\vartheta_{\text {щ }} \delta+\left(\vartheta_{\text {п }}-\vartheta_{\text {ц }}\right) \cdot \exp (1) \cdot[\operatorname{Ei}(-1)+1 / \exp (1)] \cdot \delta\right\} .
$$

Учитывая, что

$$
\operatorname{Ei}(z)=C+\ln (-z)+\sum_{r=1}^{\infty} \frac{z^{r}}{r \cdot r !} \quad \text { при }(z<0) \text { и } C=0.5772
$$

для $z=(-1)$ получим

$$
\operatorname{Ei}(-1)=C+\sum_{r=1}^{\infty} \frac{(-1)^{r}}{r \cdot r !}
$$

Учитывая, что

$$
\sum_{r=1}^{\infty} \frac{(-1)^{r}}{r \cdot r !}=-0.7966
$$

Заменим в (9) величины $\widehat{\vartheta}$ и $\vartheta_{n}$ их выражениями (10) и (11). Учитывая, что $\vartheta_{c p d}=$ const а $\vartheta_{u}$ - переменная в процессе нагрева величина, в конечном итоге получим

$$
\frac{d \vartheta_{u}}{d \tau}=\frac{a}{\delta^{2}} \cdot \frac{S_{\text {бок }} \cdot \delta}{V_{T}} \cdot \frac{\mathrm{Bi}}{1+k \cdot \mathrm{Bi}} \cdot\left(\vartheta_{c p d}-\vartheta_{u}\right)
$$

С помощью нормировки приведем уравнение (12) к обезразмеренному виду. Температуры пронормируем величиной $\vartheta_{c p d}=$ const, т.е.

$$
\bar{\vartheta}_{u}=\frac{\vartheta_{u}}{\vartheta_{c p d}}=\frac{t_{u}-t_{0}}{t_{c p d}-t_{0}}, \quad \bar{\vartheta}_{c p d}=\frac{\vartheta_{c p d}}{\vartheta_{c p d}}=\frac{t_{c p d}-t_{0}}{t_{c p d}-t_{0}}=1,
$$

а время комплексом

$$
\frac{a}{\delta^{2}} \cdot \frac{S_{6 о \kappa} \cdot \delta}{V_{T}} \cdot \frac{\mathrm{Bi}}{1+k \cdot \mathrm{Bi}} .
$$

В результате уравнение (12) запишется в виде

$$
\frac{d \bar{\vartheta}_{u}}{d \hat{H} o}=1-\bar{\vartheta}_{u}
$$

или 


\section{СТУДЕНТСЬКА НАУКА}

где

$$
\frac{d \bar{\vartheta}_{u}}{d \widehat{H}_{o}}+\bar{\vartheta}_{u}=1
$$

$$
\widehat{\mathrm{Ho}}=\frac{a \tau}{\delta^{2}} \cdot \frac{S_{\text {бок }} \cdot \delta}{V_{T}} \cdot \frac{\mathrm{Bi}}{1+k \cdot \mathrm{Bi}} \text { или } \mathrm{Ho}=\mathrm{Fo} \cdot \frac{S_{\text {бок }} \cdot \delta}{V_{T}} \cdot \frac{\mathrm{Bi}}{1+k \cdot \mathrm{Bi}}
$$

Обычно в нестационарных задачах теплообмена в качестве нормированного времени выступает число подобия $F o$, которое, также, называется числом гомохронности. По аналогии назовем выражение (14) модифицирорванным числом гомохронности. Оно помимо $F o$ включает комплекс $\frac{S_{\text {бок }} \cdot \delta}{V_{T}}$, зависящий от геометрии нагреваемого тела, а так же комплекс $\frac{\mathrm{Bi}}{1+k \cdot \mathrm{Bi}}$, зависящий от критерия $\mathrm{Bi}$ и коэффициента $k=0.4$, обусловленного выбранной формой профиля изменения температуры.

Проанализируем полученный результат. Выражение (13) является уравнением инерционного звена в ТАУ и имеет решение вида

$$
\bar{\vartheta}_{u}=1-\exp (-\hat{\mathrm{Ho}})
$$

Оно получено для случая бесконечной пластины. Но исходные предпосылки для нее такие же, как и для бесконечного цилиндра и шара. Различие проявляется только в комплексе $\frac{S_{\text {бок }} \cdot \delta}{V_{T}}$ из (14), зависящем от формы рассматриваемого тела. Определим его величину учитывая, что в случае цилиндра или шара в качестве характерного размера принимается их радиус:

- для пластины

$$
\frac{S_{\text {бок }} \cdot \delta}{V_{T}}=\frac{S_{\text {бок }} \cdot \delta}{S_{\text {бок }} \delta}=1
$$

- для бесконечного цилиндра

$$
\frac{S_{\text {бок }} \cdot R}{V_{T}}=\frac{2 \pi R L \cdot R}{\pi R^{2} L}=2
$$

- $\quad$ для шара

$$
\frac{S_{\text {бок }} \cdot R}{V_{T}}=\frac{4 \pi R^{2} \cdot R}{4 / 3 \pi R^{3}}=3 .
$$

Применим решение (15) с учетом (14), (16), (17), (18) для оценки времени окончания переходного процесса нагрева тел. Сравним полученные данные с известными результатами точных решений [2]. В ТАУ исходя из (15) переходный процесс считается завершенным при $\hat{\mathrm{H}}=3$. Это соответствует отклонению искомой величины (в данном случае $\left.\bar{\vartheta}_{u}\right)$ от максимально возможного ее значения (в данном случае 1 ) не более чем на $5 \%$, т.е. $\bar{\vartheta}_{u}=0.95$. Для этого случая из (14)

$$
\mathrm{Fo}_{1}=\frac{\hat{\mathrm{Ho}}}{\frac{S_{\text {бок }} \cdot \delta}{V_{T}} \cdot \frac{\mathrm{Bi}}{1+\mathrm{k} \cdot \mathrm{Bi}}}
$$

В [2] рассматривается охлаждение тела. Процесс нагрева и охлаждения симметричны. Будем определять $\mathrm{Fo}_{2}$ для случая $\bar{\vartheta}_{u}=0.05$. Результаты сравнения $\mathrm{Fo}_{1}$ и $\mathrm{Fo}_{2}$, а следовательно и времен окончания процесса нагрева, на основании относительной погрешности приведены в таблице 1. 
Таблица 1. Сравнение точного и приближенного расчета времени окончания процесса нагрева

\begin{tabular}{|c|c|c|c|c|c|c|c|c|c|}
\hline \multirow{2}{*}{$\mathrm{Bi}$} & \multicolumn{3}{|c|}{ Пластина } & \multicolumn{3}{|c|}{ Цилиндр } & \multicolumn{3}{|c|}{ Шар } \\
\cline { 2 - 11 } & $\mathrm{Fo}_{2},[2]$ & $\begin{array}{c}\mathrm{Fo}_{1}, \\
\text { расчет }(14)\end{array}$ & $\varepsilon, \%$ & $\mathrm{Fo}_{2},[2]$ & $\begin{array}{c}\mathrm{Fo}_{1}, \\
\text { расчет }(14)\end{array}$ & $\varepsilon, \%$ & $\mathrm{Fo}_{2},[2]$ & $\begin{array}{c}\mathrm{Fo}_{1}, \\
\text { расчет }(14)\end{array}$ & $\varepsilon, \%$ \\
\hline $\mathbf{0 , 0 0 5}$ & 600,34 & 601,2 & $\mathbf{0 , 1 4}$ & 300,06 & 300,6 & $\mathbf{0 , 1 8}$ & 200,1 & 200,4 & $\mathbf{0 , 1 7}$ \\
\hline $\mathbf{0 , 0 1}$ & 300,9 & 301,2 & $\mathbf{0 , 0 9}$ & 150,4 & 150,6 & $\mathbf{0 , 1 5}$ & 100,20 & 100,4 & $\mathbf{0 , 2 0}$ \\
\hline $\mathbf{0 , 1}$ & 31,12 & 31,2 & $\mathbf{0 , 2 6}$ & 15,48 & 15,6 & $\mathbf{0 , 7 8}$ & 10,29 & 10,4 & $\mathbf{1 , 1}$ \\
\hline $\mathbf{1 , 0}$ & 4,200 & 4,2 & $\mathbf{\sim 0}$ & 2,02 & 2,1 & $\mathbf{4 , 0}$ & 1,31 & 1,4 & $\mathbf{6 , 7}$ \\
\hline $\mathbf{1 0}$ & 1,58 & 1,5 & $\mathbf{5 , 1}$ & 0,725 & 0,75 & $\mathbf{3 , 4}$ & 0,45 & 0,5 & $\mathbf{1 0 , 2}$ \\
\hline $\mathbf{1 0 0}$ & 1,34 & 1,23 & $\mathbf{8 , 1}$ & 0,612 & 0,615 & $\mathbf{0 , 5 6}$ & 0,38 & 0,41 & $\mathbf{7 , 5}$ \\
\hline $\mathbf{1 0 0 0}$ & 1,32 & 1,2 & $\mathbf{8 , 5}$ & 0,601 & 0,602 & $\mathbf{0 , 1 3}$ & 0,37 & 0,400 & $\mathbf{7 , 3}$ \\
\hline
\end{tabular}

Коэффициент $k=0.4$ в (14) рассчитан для профиля изменения температуры (1) в пластине, но лучший результат (величина относительного отклонения) по сравнению с точным решением получен для цилиндра. Это может быть выражением того факта, что профили изменения температуры в телах различных форм имеют общий характер но, в то же время, и некоторые особенности, трудно учитываемые при расчетах. В [1] отмечено, что в этом случае для определения (уточнения) интегральных коэффициентов при сохранении общей формы аналитического выражения, могут быть использованы немногочисленные эксперименты. В их качестве в рассматриваемом случае могут быть использованы результаты точных решений. Так вместо общего значения $k=0.4$ использование его незначительных вариаций ( $k=0.42$ - для пластины, $k=0.39$ - для цилиндра, $k=0.36$ - для шара) дает результаты, приведенные в табл. 2.

Таблица 2. Сравнение точного и приближенного времени окончания процесса нагрева после корректировки коэффицинета $k$

\begin{tabular}{|l|l|l|l|l|l|l|l|l|l|}
\hline \multirow{3}{*}{$\mathrm{Bi}$} & \multicolumn{4}{|l}{ Пластина $k=0.42$} & \multicolumn{4}{l|}{ Цилиндр $k=0.39$} & \multicolumn{2}{l|}{ Шар $k=0.36$} \\
\cline { 2 - 11 } & $\mathrm{Fo}_{2},[2]$ & $\begin{array}{l}\mathrm{Fo}_{1}, \\
\text { расчет }(14)\end{array}$ & $\varepsilon, \%$ & $\mathrm{Fo}_{2},[2]$ & $\begin{array}{l}\mathrm{Fo}_{1}, \\
\text { расчет (14) }\end{array}$ & $\varepsilon, \%$ & $\mathrm{Fo}_{2},[2]$ & $\begin{array}{l}\mathrm{Fo}_{1}, \\
\text { расчет }(14)\end{array}$ & $\varepsilon, \%$ \\
\hline $\mathbf{0 , 0 0 5}$ & 600,34 & 601,26 & $\mathbf{0 , 1 5}$ & 300,06 & 300,6 & $\mathbf{0 , 1 8}$ & 200,1 & 200,4 & $\mathbf{0 , 1 5}$ \\
\hline $\mathbf{0 , 0 1}$ & 300,9 & 301,3 & $\mathbf{0 , 1}$ & 150,4 & 150,6 & $\mathbf{0 , 1 5}$ & 100,20 & 100,36 & $\mathbf{0 , 1 7}$ \\
\hline $\mathbf{0 , 1}$ & 31,12 & 31,26 & $\mathbf{0 , 5}$ & 15,48 & 15,59 & $\mathbf{0 , 7}$ & 10,29 & 10,36 & $\mathbf{0 , 7}$ \\
\hline $\mathbf{1 , 0}$ & 4,200 & 4,26 & $\mathbf{1 , 4}$ & 2,02 & 2,085 & $\mathbf{3 , 3}$ & 1,31 & 1,36 & $\mathbf{3 , 7}$ \\
\hline $\mathbf{1 0}$ & 1,58 & 1,56 & $\mathbf{1 , 3}$ & 0,725 & 0,735 & $\mathbf{1 , 3}$ & 0,45 & 0,46 & $\mathbf{1 , 4}$ \\
\hline $\mathbf{1 0 0}$ & 1,34 & 1,29 & $\mathbf{3 , 6}$ & 0,612 & 0,6 & $\mathbf{1 , 9}$ & 0,38 & 0,37 & $\mathbf{3 , 0}$ \\
\hline $\mathbf{1 0 0 0}$ & 1,32 & 1,26 & $\mathbf{3 , 9}$ & 0,601 & 0,587 & $\mathbf{2 , 3 6}$ & 0,37 & 0,36 & $\mathbf{3 , 4}$ \\
\hline
\end{tabular}

Во всем диапазоне изменения Вi для всех рассмотренных тел время окончания процесса нагрева, рассчитанное с помощью предложенной модели в сосредоточенной постановке отличается от результатов точного решения модели в распределенной постановке менее чем на 4\% т.е. соответствует допустимой точности инженерных расчетов. При этом момент окончания переходного процесса определяется единой величиной Нิо $=3$, в то время как в [2] в этом случае каждому телу и каждому значению $\mathrm{Bi}$ соответствует свое значение Fo .

Таким образом, время окончания нагрева тел, рассматриваемых форм, может быть определено с помощью одного выражения (15) и двух коэффициентов (табл. 3).

Таблица 3. Коэффициенты формы

\begin{tabular}{|l|c|c|}
\hline & $\frac{S_{\text {бок }} \cdot l}{V_{T}}$ & $k$ \\
\hline Пластина & 1 & 0,42 \\
\hline Цилиндр & 2 & 0,39 \\
\hline Шар & 3 & 0,36 \\
\hline
\end{tabular}


Кроме решения основной поставленной задачи определения момента окончания переходного процесса при нагреве тела предложенная модель позволяет оценить:

- $\quad$ значение температуры $\bar{\vartheta}_{u}$ в центре тела (15) в любой момент времени;

- $\quad$ значение температуры на поверхности тела $\bar{\vartheta}_{n}$. Для этого необходимо пронормировать с помощью $\vartheta_{c p д}$ выражение (10). В результате, с учетом (15), получим

$$
\bar{\vartheta}_{n}=\frac{k \cdot \mathrm{Bi}+(1-k) \cdot \bar{\vartheta}_{u}}{(1-k)+k \cdot \mathrm{Bi}}=1-\frac{\exp (-\hat{\mathrm{Ho}})}{1+\frac{k}{1-k} \mathrm{Bi}}
$$

- $\quad$ значение температуры в любой точке тела. Для этого необходимо пронормировать с помощью $\vartheta_{c p д ~}$ выражение (1). В результате, с учетом (15) и (20), получим

$$
\bar{\vartheta}_{x}=1-\left[1-\frac{\mathrm{Bi}}{\left(\frac{1}{k}-1+\mathrm{Bi}\right)} \cdot \exp \left(1-\frac{1}{\bar{x}}\right)\right] \cdot \exp (-\hat{\mathrm{H}} \mathrm{o}) ;
$$

- $\quad$ величину $\mathrm{Bi}$ при различном времени или момент времени при различных $\mathrm{Bi}$, начиная с которого процесс нагрева можно описать с помощью сосредоточенной модели, т.е. разность температур на поверхности и в центре тела в этом случае будет меньше наперед заданной величины, записанной, например, в относительном виде

С учетом (15) и (21) получим

$$
\frac{t_{n}-t_{u}}{t_{c p d}-t_{0}}=\frac{\left(t_{n}-t_{0}\right)-\left(t_{u}-t_{0}\right)}{t_{c p d}-t_{0}}=\frac{\vartheta_{n}-\vartheta_{u}}{\vartheta_{c p d}}=\bar{\vartheta}_{n}-\bar{\vartheta}_{u}<f
$$

$$
\bar{\vartheta}_{n}-\bar{\vartheta}_{u}=\frac{\mathrm{Bi}}{\left(\frac{1}{k}-1+\mathrm{Bi}\right)} \cdot \exp (-\hat{\mathrm{H}})<f
$$

Так, например, величина $f=0.1$ говорит о том, что при заданных теплофизических свойствах тела и условиях теплообмена с окружающей средой в рассматриваемый момент времени разность температур на поверхности и в центре нагреваемого тела не превосходит $10 \%$ от величины $t_{c p d}-t_{0}$.

\section{Заключение}

Единое решение в виде (15) для тел различной формы удалось получить в следствии:

- перехода к модели в сосредоточенной постановке;

- перехода от критериев подобия к числам подобия при записи модели в обезразмеренном виде. Такой переход позволяет уменьшить количество переменных, определяемых $\pi$-теоремой (теоремой Букингема) на количество критериев подобия.

Так решение рассматриваемой задачи в [2] зависит от обезразмеренного времени в виде числа подобия Fо и критерия подобия $\mathrm{Bi}$, т.е. $2^{\mathrm{x}}$ переменных. Поэтому в графическом виде для каждой формы тела и рассматриваемой точки в его пространстве решение изображается в виде набора кривых. В предложенном решении (15) искомая величина зависит лишь от обезразмеренного времени в виде модифицированного числа гомохронности Но и в графическом виде может быть отображено в виде одной кривой.

Выражение (15) справедливо для различных рассмотренных форм тел. Это дает основание для предположения о его справедливости и для произвольной формы тела. При этом значения коэффициента $k$ и комплекса $\frac{S_{\text {бок }} \cdot R}{V_{T}}$ могут быть получены, как отмечалось выше, из немногочисленных экспериментов. Подтверждение последнего тезиса может служить основой для дальнейшей работы. 